\title{
A Novel Two Surgeons Approach to Bronchial Foreign Body Removal by Optical Instruments- An 11 Years Institutional Experience
}

\author{
Abhik Sikdar $^{1}$ - Avani Mahajan ${ }^{1} \cdot$ Sameer Nivsarkar $^{1} \cdot$ Shrikant Phatak $^{1}$. \\ Richa Agarwal ${ }^{1}$
}

Received: 25 June 2021 / Accepted: 31 August 2021/Published online: 12 September 2021

(C) Association of Otolaryngologists of India 2021

\begin{abstract}
A potentially life-threatening emergency in ENT practice is the aspiration of tracheobronchial foreign body. This commonly affects young children and requires early extraction. Successful removal provides immediate relief. However procedural complications can cause devastating morbidity like anoxic encephalopathy or instant death. The aim of this study is to describe the experience in our institution with two surgeons approach for removal of tracheobronchial foreign bodies by telescope and optical forceps. This is an observational study from the period of March 2010 to April 2021 in which 96 patients (59 males, 37 females; age range 3 months to 15 years) were diagnosed with foreign body aspiration. In all cases rigid bronchoscopy and FB removal under general anaesthesia was performed using the two surgeons technique. This technique was instituted once Storz make paediatric telescope (2.9 mm diameter $36 \mathrm{~cm}$ length), optical grasping forceps and optical suction were procured in March 2010. Aspirated material was inorganic or organic with varying location in right main bronchus, left main bronchus or the trachea. We highlight the OT setup, surgical algorithm and the advantages of this technique. Visual and tactile coordination between the two surgeons being the crux of this procedure; allows hands on mentoring of the next generation of budding surgeons in a real-life scenario without compromising patient safety.
\end{abstract}

Abhik Sikdar

abhik_sikdar@yahoo.co.in

1 Department of ENT, Choithram Hospital and Research Center, Flat No 902, Block A3, Avasa Housing, opp Phalbag, AB Road, Indore, MP 452012, India
Keywords Two surgeons technique .

Foreign Body Aspiration (FBA) - Rigid bronchoscope . Telescope $\cdot$ Optical forceps

\section{Introduction}

High incidence of foreign body aspiration (FBA) in infants and younger children is common as toddlers use their mouth to explore their surroundings. This can lead to high mortality and morbidity if it is not diagnosed and managed in time $[1,2]$. The most common types of FBA in children are seeds, peanuts, food particles, and toys. Items such as toy parts, paper clips, pins, and pen caps are usually noticed to be aspirated in older children [3, 4]. The bronchial FB tend to get lodged in the straighter right main bronchus. Airway mucosal inflammation and oedema is seen on aspiration of organic FB. Late diagnosis causes granulation at obstruction site with recurrent or persistent pneumonia.

Our goal is to highlight the life-threatening nature of bronchial FBA and to discuss the novel therapeutic intervention method of two surgeons approach to FB removal by optical instruments. During extraction by optical instruments live visualisation occurs with all members of surgical team participating in the decision making process. This helps in developing anticipation for instrument handling between the surgical team members. Thus, there is sharing of experience in a real-life scenario without compromising patient safety. 


\section{Materials and Method}

This observational study included 96 children aged less than 15 years, diagnosed with tracheobronchial FBA between March 2010 and April 2021. After accessing all records from Medical Records Department, retrospective analysis was made of the patient age, gender, FB characteristics, location and clinical outcomes.

Provisional diagnosis of FBA was made on the basis of detailed history with emphasis on any witnessed aspiration and intractable cough. Auscultatory examination of unequal air entry and unequal breath sounds was pathognomic, but was not present in all cases. Radiological investigation CT chest (68) or XRAY chest (28) was done for FB screening. We counselled and took informed consent regarding chances of tracheostomy, mechanical ventilation, repeat endoscopy and cardiac arrest. If patient was already on mechanical ventilation preoperatively, then pupillary reflex was checked to document any pre-existing anoxic encephalopathy.

When FBA was diagnosed from clinical and radiological evaluations, rigid bronchoscopy was performed under general anaesthesia with the jet ventilation. All foreign bodies were extracted using paediatric rigid ventilating bronchoscope with telescope and optical instruments using the two surgeons technique.

\section{OT Setup}

Appropriately sized Macintosh laryngoscope was used to visualise the laryngeal inlet to guide in the rigid ventilating bronchoscope. Appropriately sized Magills forceps was kept ready to remove FB if it slipped out of optical forceps in the oral cavity or pharynx. Tracheostomy set and pre sterile packed tracheostomy tube was kept ready in OT. Instrument assembly is described in Fig. 1.

(1) In our setup we use appropriate size rigid ventilating bronchoscope. Size is calculated as in Table 1:

(2) Telescope 0 degree, $2.9 \mathrm{~mm}$ diameter, $36 \mathrm{~cm}$ length.

(3) Optical instruments: pincer, rat tooth and peanut forceps (Fig. 1C.a, b, c respectively).

(4) Telescope mounted suction and plain suction (telescope mounted suction fits only in bronchoscope size 4 and above).

(5) Tracheostomy set and appropriately sized tracheostomy tube.

(6) Bronchoscope prism (Fig. 1A.e) 2 xenon light sources, 2 light cables (Fig. 1A.a, b).

(7) Endoscopic camera, medical grade monitor and recording system.

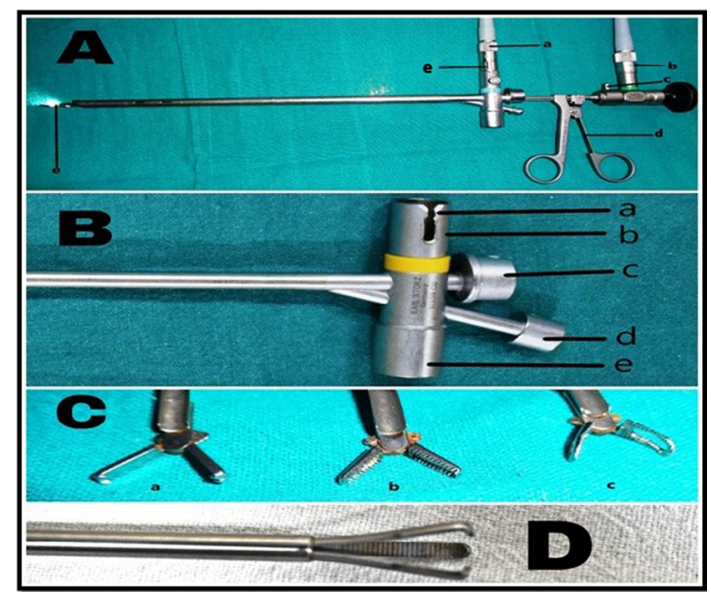

Fig. 1 Aa light cable 1, Ab light cable 2, Ac forceps lock, Ad spring action forceps handle, Ae bronchoscope prism, Ba distal prism slot, $\mathbf{B b}$ proximal prism slot, $\mathbf{B c}$ telescope slot, $\mathbf{B d}$ jet ventilator slot, $\mathbf{B e}$ anaesthesia circuit slot, $\mathbf{C a}$ pincer forceps, $\mathbf{C b}$ rat tooth forceps, $\mathbf{C c}$ peanut forceps, D Uteroscopic tripronged forceps

Detailed positioning in this novel two surgeons technique as in Fig. 2

(a) The rigid bronchoscope introduced by Surgeon 1 who sits on head end of the table.

(b) Surgeon 2 who introduces the telescope mounted optical instruments, stands behind surgeon 1. This allows both to look directly to the monitor and allows easy instrumentation without fouling with each other's hand.

(c) Monitor.

(d) Primary nurse besides monitor and bronchoscope trolley.

(e) Circulatory nurse with tracheostomy set stands at patient foot end.

(f) Anaesthesia workstation.

(g) Anaesthetist with workstation on right of patient head end.

\section{Procedure Algorithm}

(1) Assistant checks focus of camera after mounting on telescope, completes white balance and zooms in appropriately (telescope is $2.9 \mathrm{~mm}$ diameter as opposed to $4 \mathrm{~mm}$ of nasal endoscope).

(2) Pre oxygenation done with bag mask ventilation or through endo tracheal tube if pre-intubated.

(3) 2\% Lignocaine with1: 200,000 Adrenaline injected over neck to allow for rapid bloodless access in case of emergency tracheostomy.

(4) After visualising laryngeal inlet with Macintosh anaesthetic laryngoscope, rigid ventilating bronchoscope is inserted into larynx in a pen holding 
Table 1 Size of bronchoscope according to age

\begin{tabular}{ll}
\hline Age & Bronchoscope size \\
\hline Term newborn & 3.0 \\
6 months & 3.0 \\
1 year & 3.5 \\
2 year & 3.5 \\
3 year & 4.0 \\
4 year & 4.5 \\
5 year and above & 5.0 \\
\hline
\end{tabular}

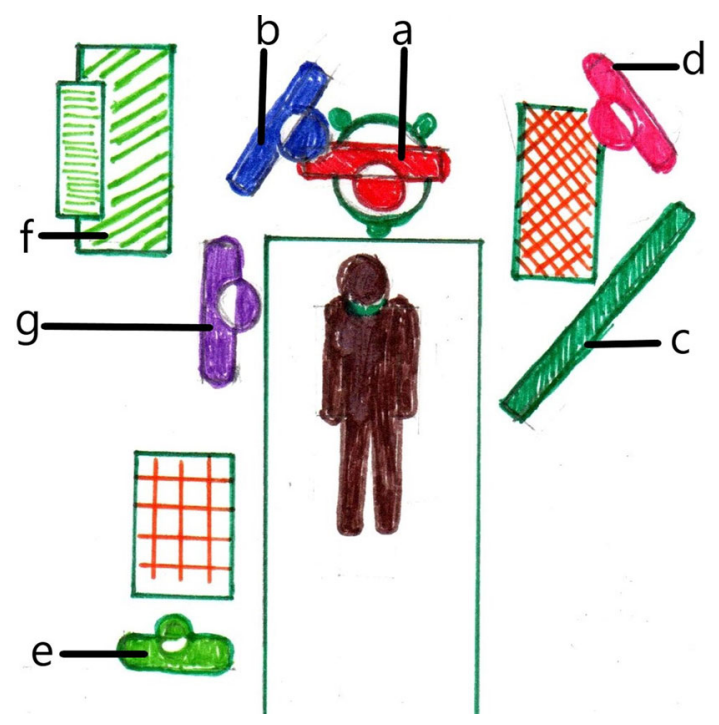

Fig. 2 a Surgeon 1 sitting, b Surgeon 2 standing, c Monitor, d Primary nurse, e Circulatory nurse, f Anaesthesia workstation, g Anaesthetist

fashion by surgeon 1 sitting on head end. The bronchoscope must be rotated at right angles so that the bevel faces laterally, allowing easy insertion through the glottic chink. Otherwise, the scope tends to slip posteriorly into the oesophagus. Jet ventilation is started as soon as bronchoscope is in the trachea.

(5) Once bronchoscope reaches the carina, patient's head is turned slightly to opposite side to allow easy insertion into intended bronchus. If required suction and clearance of secretions is done for better visualisation.

(6) After surgeon 1 visualises FB with naked eye through proximal illumination of prism, surgeon 2 standing behind introduces telescope through end on opening (Fig. 1B.c) of bronchoscope to confirm nature of $\mathrm{FB}$.
(7) Optical forceps is chosen according to nature of FB. Peanut in case of soft $\mathrm{FB}$ and rat tooth or pincer in case of hard FB (Fig. 1C).

(8) Telescope is then withdrawn and optical forceps mounted onto it. The light cable on telescope (Fig. 1A.b) has to be rotated vertically up to $12 \mathrm{o}$ clock so as to lock the forceps (Fig. 1A.c) onto the telescope, keeping the spring action forceps handle at 6 o clock (Fig. 1A.d).

(9) While inserting the telescope mounted forceps into the bronchoscope, the proximal illuminating prism has to be pulled outside (Fig. 1B.b-B.a) to allow space for insertion.

(10) Jet ventilation which is done through the thin side slot (Fig. 1B.d) is stopped during extraction as the moist air coming out of bronchus tends to fog the scope. Strict watch is kept on saturation and when required bronchoscope is removed to allow for dedicated bag-mask ventilation. In absence of Jet ventilation equipment, the standard ventilating anaesthesia circuit may be connected to the large side port (Fig. 1B.e).

(11) Surgeon 1 stabilises bronchoscope prevents it from rotating due to weight of optical cable attached to prism (Fig. 1B.b).

(12) While inserting the telescope mounted forceps into bronchoscope and reaching the FB, surgeon 2 must take care that there is no bending of the delicate optical system. Any bend of the system shows up as crescentic darkening along the sides of the round image (Fig. 3a). This has to be corrected immediately else there is permanent instrument damage.

(13) Once the forceps tip reaches near FB, the bronchoscope has to be pulled back a few millimetres to allow the jaws to open completely. Sometimes the jaws are opened just proximal to impacting FB to dilate the bronchus and create forceps space. Care is taken to prevent pushing smooth FB distally when closing jaw. To ensure this the jaws of a peanut forceps has to go beyond the maximum diameter of a smooth FB (Fig. 3b). However, a rat tooth forceps attains a firm grip on a hard and rough FB even when holding it partially.

(14) The Storz forceps handle has spring action to prevent slipping of FB without crushing it (Fig. 1A.d).

(15) Where possible the FB is pulled within the bronchoscope. Else it is pulled out maximally till the jaws of the optical forceps assembly touches the bronchoscope tip.

(16) The forceps and the bronchoscope are held in tight contact by the two surgeons by visual feedback from monitor. 


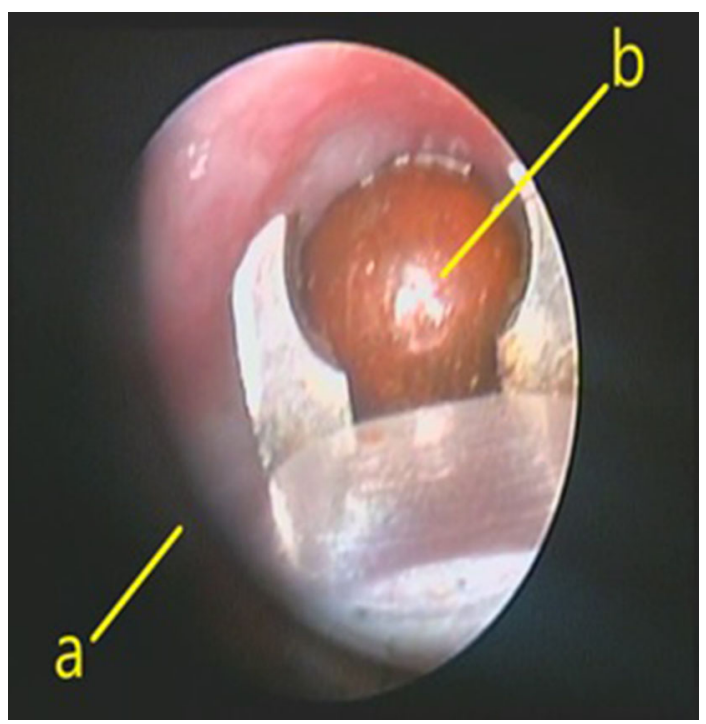

Fig. 3 a crescentic darkening, b smooth FB

(17) Near the cords the bronchoscope is rotated anteriorly for the bevel to maximally open the glottis and prevent glottic spasm. This prevents slippage of FB. FB slipping into the subglottis may cause near total airway obstruction and needs forceful jet ventilation to dislodge distally it into a bronchus.

(18) Check bronchoscopy is done to remove any trapped discharge beyond erstwhile obstruction and to remove any remnant FB.

(19) Preferentially patient is extubated on table.

(20) Rarely in cases of severe pulmonary oedema or pneumonia patient is managed in intensive care on ventilator and extubated later.

Crux of our technique is the visualisation and coordination between the two surgeons. Surgeonl concentrates to stabilise the bronchoscope and prevents rotation by the weight of the attached light cable. In neonate for suctioning the plain suction is introduced along with the telescope by surgeon 2 using both hands. This is required as a telescope mounted suction is too thick to enter a neonate bronchoscope of size 3. In one case, aspirated marble was too big for the jaws of the optical forceps to go beyond its maximum diameter. Here surgeonl held the bronchoscope while surgeon 2 introduced the telescope and ureteric tripronged forceps together (Supplementary video1). The ureteroscopic tripronged forceps opened wider than the marble (Fig. 1D) and its thin shaft allowed insertion alongside telescope within bronchoscope.

\section{Results}

Our experience- total of 96 FBs were diagnosed with FBA between March 2010 and April 2021 which have been successfully extracted by two surgeons technique.

\section{Age and Sex Distribution Table 2}

59 males $(61.5 \%), 37$ females $(38.5 \%)$; range 3 months15 years

\section{Location of FB-}

Lower trachea- $4(4.2 \%)$ carina $6(6.3 \%)$, right bronchus$59(61.4 \%)$ left bronchus-27(28.1\%).

\section{Type of FB in Different Years Table 3}

Organic FBs were 74(77.1\%) while inorganic was 22(22.9) in the last 11 years.

The most common inorganic FB were beads followed by plastic decorative material and broken toy pieces. Unusual inorganic FB removed were 1 marble, 1 screw (supplementary video 2), 1 pen cap, 2 whistles and 3 led bulbs (supplementary video3). The most frequently acquainted organic FB was peanut followed by a beetle nut and custard apple seed (supplementary video 4 ).

\section{Peri Operative and Post-Operative Experience-}

All FB were successfully removed with no need for redo bronchoscopy. No case required tracheostomy. There was no death on table.19 out of 96 patients (20\%) were on mechanical ventilation before they could be shifted to OT. Post bronchoscopy 14 patients (14\%) were not extubated on table of which 9 were preoperatively on ventilator. These postop ventilated patients had severe pulmonary oedema, pneumonia or excess granulations at the site of old FB.13 out of these 14 were extubated within $48 \mathrm{~h}$. The remnant case was preoperatively on ventilator with anoxic encephalopathy and had dilated and sluggish pupillary reaction. Here the FB of custard seed was successfully extracted but the boy succumbed on post-operative day 4 due to neurologic complications. 82 (86\%) patients including 10 who were preoperatively on ventilator did not require postoperative mechanical ventilation. 
Table 2 Age and sex distribution of patients

\begin{tabular}{lrcr}
\hline Age & Male & Female & Total \\
\hline $0-1$ & 3 & 2 & 5 \\
$1-2$ & 8 & 5 & 13 \\
$2-3$ & 19 & 12 & 31 \\
$3-4$ & 10 & 8 & 18 \\
$4-5$ & 7 & 4 & 11 \\
$5-6$ & 5 & 2 & 7 \\
$6-7$ & 3 & 0 & 3 \\
$7-8$ & 0 & 1 & 1 \\
$8-9$ & 0 & 0 & 0 \\
$9-10$ & 1 & 1 & 2 \\
$10-11$ & 1 & 1 & 2 \\
$11-12$ & 1 & 1 & 2 \\
$12-13$ & 0 & 0 & 0 \\
$13-14$ & 0 & 0 & 0 \\
$14-15$ & 1 & 0 & 1 \\
Total & 59 & 37 & 96 \\
\hline
\end{tabular}

Table 3 Types of FB removed in different years

\begin{tabular}{|c|c|c|c|}
\hline Year & Number & Organic & Inorganic \\
\hline 2010 & 7 & 5 & 2 \\
\hline 2011 & 6 & 5 & 1 \\
\hline 2012 & 12 & 9 & 3 \\
\hline 2013 & 7 & 7 & 0 \\
\hline 2014 & 10 & 8 & 2 \\
\hline 2015 & 12 & 9 & 3 \\
\hline 2016 & 6 & 5 & 1 \\
\hline 2017 & 14 & 10 & 4 \\
\hline 2018 & 9 & 5 & 4 \\
\hline 2019 & 3 & 2 & 1 \\
\hline 2020 & 6 & 5 & 1 \\
\hline 2021 till April & 4 & 4 & 0 \\
\hline Total & 96 & 74 & 22 \\
\hline
\end{tabular}

\section{Discussion}

The most frequently encountered life-threatening emergency in ENT practice is FBA. It may not be immediately diagnosed because there are no specific clinical manifestations. Witnessed history of FBA and choking is extremely suggestive but rarely encountered. The classical clinical presentation of coughing, wheezing and diminished air inflow, is seen in less than $40 \%$ of the patients. Other symptoms include cyanosis, fever and stridor [5]. FBA in children may present with respiratory symptoms days or months after aspiration has occurred [6]. Rarely FBA can be completely asymptomatic. A study by Mundra et al. postulates any child presenting with cough, stridor and respiratory distress of acute onset should be suspected for FBA unless proved otherwise [7]. Of patients with laryngotracheal foreign bodies, $67 \%$ experience associated complications when removed after delay of more than $24 \mathrm{~h}$ [7].

Saki discussed that in different studies males were more affected (60-66\%), specially more in the first and second year of life [5]. Other studies cite that FBA maximally affects children aged $2-5[8,9]$. In our study, this age group accounted for more than $62.5 \%$ of the patients. In our study $61.5 \%$ were males and $38.5 \%$ were females. Korlacki et al. stated that the most commonly aspirated foreign bodies in paediatric age were nuts and sunflower seeds (about 68\% of cases) [10]. Fidkowski et al. stated $81 \%$ of the aspirated foreign bodies were organic materials with the most commonly aspirated foreign bodies reported as nuts (especially peanuts) and seeds (mainly sunflower and watermelon) [11]. However, an Italian series that found tooth to be the most commonly aspirated object (33/128) [12]. In our study $74(77.1 \%)$ were organic foreign bodies while $22(22.9 \%)$ inorganic. In our series the most frequently acquainted organic FB was peanut followed by a beetle nut and custard apple seed. Organic foreign bodies cause surrounding tissue reaction as they enlarge in size when they absorb water. This converts a partial airway obstruction to a complete one. Delayed removal is related to higher rate of complications. Mucolytics, parenteral antibiotics and chest physiotherapy help in prevention of pneumonia and shorten the hospital stay. Our data regarding the gender, age and nature of FBA is consistent with published literature.

We encountered total 96 FBAs in which lower trachea$4(4.2 \%)$ carina $6(6.3 \%)$, right bronchus-59(61.4\%) left bronchus-27(28.1\%). Saki et al. noted the most common site of foreign body lodgement as right main bronchus in $560(55.1 \%)$ patients followed by left main bronchus in 191 (18.8\%) [5]. In another study by Panda et al. the most common site of FB was right main bronchus in 112 (64.74\%) patients, followed by left main bronchus in 44 $(25.43 \%)$ and carina in $17(9.83 \%)$ cases [13].

Computed tomography (CT) chest helps in accurate diagnosis of FBA including its nature and site. Chest $\mathrm{X}$-rays though specific has a poor sensitivity value as FBA diagnostic tool. Thoracic computed tomography (CT) with virtual bronchoscopy (reformatted 3-dimensional CT image that generates intraluminal views of the airway bronchi) is an emerging modality. However, mucus plug, tumours, or other obstructive lesions can produce falsepositive findings. In our observational study, CT chest correctly identified all 68 children with FBA. Only Xray chest was done in the rest 28 patients who had strong 
suggestive history and were either too poor or too sick to be shifted to CT console. CT and virtual bronchoscopy are more sensitive diagnostic modalities for foreign body aspiration in comparison with conventional chest radiography [14].

The gold standard for diagnosis and management of paediatric FBA is rigid ventilating bronchoscopy and removal. Our study highlights the usage of rigid bronchoscope and telescope using the two surgeons technique. Close communication between the anaesthesiologist, both surgeons, assistants and the nursing staff is mandatory. We also inject 2\% lignocaine with 1:200,000 adrenaline in neck to allow for rapid bloodless access in case of emergency tracheostomy. In study by Hasridaz from 1987 to 2005, of the 1035 children taken for bronchoscopy, laryngeal oedema, laryngeal spasm or bronchospasm with insufficient ventilation were observed in 37 patients. While 11 of these patients recovered with bronchodilator treatment, 26 required mechanical ventilation support for up to $48 \mathrm{~h}$. Bleeding was observed in 6 patients during bronchoscopy which was stopped with washing and aspiration [15]. In another study by Panda, mechanical ventilation was required in $13(7.51 \%)$ cases in the pre-operative period and $20(11.56 \%)$ cases in the post-operative period. Redo bronchoscopy was done in $7(4.05 \%)$ cases for retained FB. Mortality was seen in $04(2.31 \%)$ cases, 03 died pre-operatively while 01 died in the post-operative period [13]. In our study 19 (20\%) required preoperative mechanical ventilation.14 (14\%) patients required postoperative mechanical ventilation including 9 who were shifted into OT pre-intubated. Failure of extubation was due to severe pulmonary oedema, pneumonia or excess granulations at site of old FB. Bleeding from granulation during check bronchoscopy was noted in 3 patients and was successfully stopped on table with adrenaline wash. No case required tracheostomy. All FBA were successfully removed without redo bronchoscopy. There was no on table mortality. One patient died on post op day 4 due to preoperative anoxic encephalopathy. The overall mortality of our study is $1 \%$.

Complications of FBA can include severe laryngeal oedema, bronchospasm, atelectasis, pneumonia, lung abscess, pneumothorax, pulmonary oedema, bronchial haemorrhage and airway obstruction requiring tracheotomy or reintubation. The evolution of FBA can lead to variable degrees of respiratory distress, atelectasis, chronic coughing, recurrent pneumonia, and even death [16, 17]. Prolonged hypoxia during removal may cause morbid complications like cardiac arrest and irreversible anoxic encephalopathy. No patient in our series had such procedural complications during OT.

Study by Dubey et al. stated that optical forceps have advantage of high resolution \& magnified view of airway while spring action in forceps handle allowed better tactile sensation. These helped in correctly identifying the type, size and site of tracheobronchial foreign body [18]. Our patients were similarly benefitted from optical instrumentation.

This study pioneers the novel two surgeons technique of using the rigid bronchoscope and optical forceps for saving lives of choking children. No comparative data of two surgeons technique available in literature.

\section{Conclusion}

For paediatric FBA, rigid bronchoscopy along with optical instruments is the procedure of choice as it allows excellent visualisation with good ventilation and airway control. We present a pioneering 11 year study in a tertiary care centre of a novel two surgeons technique for removal of FBA. We focus on the interchanging roles of two surgeons under continuous monitoring by the entire team. This procedure is associated with less chances of panic and a faster learning curve with both tactile and visual feedback. Our complication rate is comparable to published literature. The major advantage is that it allows hands on mentoring of the next generation surgeon in real life scenario without compromising patient safety.

Funding Not applicable.

Availability of data and materials Data collected from medical records department and data transparency maintained.

\section{Declarations}

Conflict of interest The authors hereby declare that there are no potential conflict of interest with respect to research and publication of this article.

\section{References}

1. Darrow DH, Holinger LD (2002) Foreign bodies of the larynx, trachea and bronchi. In: Bluestone CD, Stool SE, Apler CM, Arjmand EM, Casselbrant ML, Dohar JE et al (eds) Paediatric otolaryngology, 4th edn. WB Saunders Company, Philadelphia, PA, pp 1543-1557

2. Kaur K, Sonkhya N, Bapna AS (2002) Foreign bodies in the tracheobronchial tree: a prospective study of fifty cases. Indian $\mathrm{J}$ Otolaryngol Head Neck Surg 54(1):30-34. https://doi.org/ 10.1007/BF02911002.PMID:23119848;PMCID:PMC3450697

3. Tan HK, Brown K, McGill T, Kenna MA, Lund DP, Healy GB (2000) Airway foreign bodies (FB): a 10-year review. Int J Pediatr Otorhinolaryngol 56(2):91-99. https://doi.org/10.1016/ s0165-5876(00)00391-8 (PMID: 11115682)

4. Rimell FL, Thome A Jr, Stool S et al (1995) Characteristics of objects that cause choking in children. JAMA 274(22):17631766. https://doi.org/10.1001/jama.1995.03530220029027 
5. Saki N, Nikakhlagh S, Rahim F, Abshirini H (2009) Foreign body aspirations in infancy: a 20-year experience. Int J Med Sci 6(6):322-328. https://doi.org/10.7150/ijms.6.322.PMID:1985 173;PMCID:PMC2764343

6. Taksande A, Vilhekar K, Tyagi V (2010) Uncommon foreign body aspiration in infant. Calicut Med J. 8(2):e8

7. Mundra RK, Agrawal R, Sinha R (2014) Unusual foreign body aspiration in infants below 6 months of age. Indian J Otolaryngol Head Neck Surg 66(2):145-8. https://doi.org/10.1007/ s12070-013-0668-0 (PMID: 24822152; PMCID: PMC4016347)

8. Chen LH, Zhang X, Li SQ, Liu YQ, Zhang TY, Wu JZ (2009) The risk factors for hypoxemia in children younger than 5 years old undergoing rigid bronchoscopy for foreign body removal. Anesth Analg 109(4):1079-1084. https://doi.org/10.1213/ ane.0b013e3181b12cb5 (PMID: 19762735)

9. Brkić F, Umihanić S (2007) Tracheobronchial foreign bodies in children. Experience at ORL clinic Tuzla, 1954-2004. Int J Pediatr Otorhinolaryngol 71(6):909-15. https://doi.org/10.1016/ j.ijporl.2007.02.019 (Epub 2007 Apr 3 PMID: 17408756)

10. Korlacki W, Korecka K, Dzielicki J (2011) Foreign body aspiration in children: diagnostic and therapeutic role of bronchoscopy. Pediatr Surg Int 27(8):833-7. https://doi.org/10.1007/ s00383-011-2874-8 (Epub 2011 Mar 13. PMID: 21400033; PMCID: PMC3136687)

11. Fidkowski CW, Zheng H, Firth PG et al (2010) The anesthetic considerations of tracheobronchial foreign bodies in children: a literature review of 12,979 cases. Anesth Analg 111(4):1016-25. https://doi.org/10.1213/ANE.0b013e3181ef3e9c (Epub 2010 Aug 27 PMID: 20802055)

12. Divisi D, Di Tommaso S, Garramone M, Di Francescantonio W, Crisci RM, Costa AM, Gravina GL, Crisci R (2007) Foreign bodies aspirated in children: role of bronchoscopy. Thorac Cardiovasc Surg 55(4):249-252. https://doi.org/10.1055/s-2006924714 (PMID: 17546556)
13. Panda SS, Bajpai M, Singh A, Baidya DK, Jana M (2014) Foreign body in the bronchus in children: 22 years experience in a tertiary care paediatric centre. Afr J Paediatr Surg 11(3):252-5. https://doi.org/10.4103/0189-6725.137336 (PMID: 25047319)

14. Hong SJ, Goo HW, Roh JL (2008) Utility of spiral and cine CT scans in pediatric patients suspected of aspirating radiolucent foreign bodies. Otolaryngol Head Neck Surg 138(5):576-580. https://doi.org/10.1016/j.otohns.2007.12.039 (PMID: 18439461)

15. Hasdiraz L, Oguzkaya F, Bilgin M, Bicer C (2006) Complications of bronchoscopy for foreign body removal: experience in 1,035 cases. Ann Saudi Med 26(4):283-7. https://doi.org/10. 5144/0256-4947.2006.283 (PMID: 16883083; PMCID: PMC6074507)

16. Sirmali M, Türüt H, Kisacik E, Findik G, Kaya S, Taştepe I (2005) The relationship between time of admittance and complications in paediatric tracheobronchial foreign body aspiration. Acta Chir Belg 105(6):631-4. https://doi.org/10.1080/00015458. 2005.11679791 (PMID: 16438074)

17. Oliveira CF, Almeida JF, Troster EJ, Vaz FA (2002) Complications of tracheobronchial foreign body aspiration in children: report of 5 cases and review of the literature. Rev Hosp Clin Fac Med Sao Paulo 57(3):108-11. https://doi.org/10.1590/s0041-878 12002000300005 (PMID: 12118268)

18. Dubey NK, Upadhyay A, Raghuwanshi N et al (2021) Titleoptical forceps: the real boon for surgeon, residents and patients with foreign body aspiration. Indian $\mathbf{J}$ Otolaryngol Head Neck Surg. https://doi.org/10.1007/s12070-021-02596-8

Publisher's Note Springer Nature remains neutral with regard to jurisdictional claims in published maps and institutional affiliations. 